\title{
Impact of pool training on balance of elderly individuals.
}

\author{
Thyciane Mendonça de Andrade1, Tathiana Maria Silva Rufino², Heleodório Honorato dos Santos², \\ José Jamacy de Almeida Ferreira², Adriana Carla Costa Ribeiro Clementino², Palloma Rodrigues de Andrade ${ }^{2}$
}

\begin{abstract}
Objective: The aim of this study was to evaluate the impact of therapeutic pool training on static and dynamic balance of sedentary elderlies. Method: 10 elderly individuals took part in the study (63.10 \pm 2.33 years), and they were submitted to 24 sessions of intervention, twice a week. The assessment tools were the Balance System, Timed Up and Go Test (TUGT) and the Berg Balance System (BBS). Results: The results showed that there was a significant decrease in the global postural stability, anterior/posterior and medial/lateral scores evaluated in the dynamic platform, as well as the static global postural stability score, reduction in time to perform the TUGT and increase in the Berg Balance System score. However, no significant differences were verified in the Postural Stability tests (anterior/posterior and medial/lateral with the static platform) and in the Fall Risk. Conclusion: The regular physical training in therapeutic pool can provide sedentary elderly individuals with a significant improvement in dynamic balance.
\end{abstract}

Key words: postural stability; accidents due to falls; hydrotherapy; balance system.

\section{INTRODUCTION}

The increase in life expectancy is a global reality, even in poorer countries. ${ }^{(1)}$ Data from the Instituto Brasileiro de Geografia e Estatística (IBGE, 2010)(2) show that Brazil has been experiencing a great impact in its age structure due to population aging. Taking into account this new demographic pattern, it is important to identify factors which contribute to aging with good quality of life.

Human aging generates a higher prevalence of chronic-degenerative diseases, compromising individuals' independence and autonomy, what can result in incapacity, fragility, hospitalization and institutionalization, ${ }^{(3)}$ in addition to associated problems, such as functional decline and balance deficit $^{(4)}$ which cause a reduction in postural and gait skills, and may lead to falls.

Falls are between the main external causes of mortality in elderly individuals and are considered a public health issue, becoming an important fact with repercussions on the economy, health, and social and family relationships. ${ }^{(5-7)}$

Keeping balance during stance is essential to perform daily activities. ${ }^{\left({ }^{8}\right)}$ In order to control balance it is necessary to keep the gravity center on the base of support during static and dynamic events. ${ }^{(9)}$ The practice of physical activities may help to improve balance deficit in senescence, although people older than 60 may present some limitation when it comes to ground physical activities, besides being more susceptible to falls.

Therefore, the aquatic environment is ideal for the practice of physical activities with more safety and effectiveness, once balance training in the water is considered safe and effective in the process of rehabilitation of the elderly individual, since water acts on the musculoskeletal disorders at the same time it challenges the static and dynamic balance. In the aquatic environment, the resistance which depends on the speed of the body part that moves causes turbulence, leading to a constant search for balance and imposing a certain increase in the demand for keeping the upright posture. ${ }^{(10)}$

On the other hand, floating reduces the feeling of weight, allowing less discharge on the joints, minimizing pain, and promoting, thus, greater self-confidence and less fear of falling. In addition to the fact that floating slows the movement, resulting in increase in the sensorial feedback, it provides the

Corresponding author: Palloma Rodrigues de Andrade. Street Juiz João Agrícola Montenegro, 105 Apt N702. Brisamar, CEP: 58032210, João Pessoa (PB), Brazil. Telephone: +55 (83) 3226-9523. E-mail: pallomandrade@gmail.com.

1 Post Graduate Program in Physical Therapy by Universidade Federal de Pernambuco (UFPE), Recife (PE), Brazil.

Full list of author information is available at the end of the article.

Financial support: This work have financial support from National Council for Scientific and Technological Development of the Brazil (CNPq - Universal Edict 2013/2014).

Submission date 22 January 2016; Acceptance date 28 March 2016; Publication online date 15 April 2016 
individual with more time to go back to the correct posture after the body having unbalanced. ${ }^{(10-12)}$ However, the hydrotherapeutic resources to treat balance deficits have only become the subject of scientific studies very recently, but literature is still scarce in verifying the hydrotherapy effects on static and dynamic balance in sedentary elderly people.

In this context, balance deficit treatment becomes essential to these subjects in order to develop prevention, assistance and rehabilitation strategies, aiming to keep the autonomy and preserving the independence of the elderly individual. The aim of this study was to evaluate the aquatic training effects on the static and dynamic balance on sedentary elderly individuals.

\section{METHOD}

\subsection{Study type}

This is a prospective longitudinal study of the non-controlled clinical trial type, carried out in the Physiotherapy Clinical School in the Universidade Federal da Paraiba (UFPB). It was approved by the Research Ethics Committee in the Health Science Center of UFPB under the protocol n. 044/13. CAAE: 11565413.3.0000.5188 and followed the Declaration of Helsinki. The study was registered with www.ensaiosclinicos.gov.br (RBR-4j428f).

\subsection{Subjects}

The subjects were selected through intentional sampling from the waiting list of the Physiotherapy Clinical School in UFPB (Figure 1) according to the inclusion criteria: healthy individuals from both sexes, 60 years old or older, sedentary, ${ }^{(13)}$ independents in the gait and daily activities and that did not show any dysfunction in the neurological and cardiovascular systems, cognitive alterations (Mini Mental State Examination - MMSE $\geq 24$ ), diagnosis of vertigo, hearing problems or urinary infection.

10 elderly individuals took part in the study, from both genders ( 7 women and 3 men), who were $63.10 \pm 2.33$ years old; $69.57 \pm 6.83 \mathrm{~kg} ; 1.57 \pm 0.07 \mathrm{~m}$ and $\mathrm{BMI}$ of $28.34 \pm 1.58 \mathrm{~kg} / \mathrm{m}^{2}$. The volunteers invited to take part in the study, after having read the Informed Consent Term, were informed about the goals and procedures, and signed agreeing with their voluntary participation in the study, according to the Resolution 466/2012 of the Conselho Nacional de Saúde (CNS).

After the evaluation, the properly triaged volunteers who were according to the study started their participation in the therapeutic pool training, being assessed again in two different moments: after the twelfth (Post12) and the twenty-fourth session (Post24).

\subsection{Procedures}

\section{Evaluation}

The initial evaluation was done in the Human Movement Research Center in UFPB and consisted of the application of the MMSE and a semi-structured questionnaire with social-demographic and clinic data. Then, the subjects were submitted to the evaluation of static and dynamic balance and fall risks analysis.

Balance was evaluated through three tools: the platform Balance System - BS, ${ }^{(14)}$ the Timed Up \& Go Test (TUGT) and the Berg Balance System - BBS (Brazilian version); the Fall Risk was measured by the BS score.

The postural stability and fall risk evaluation was measured by the Balance System (BS) which consists of a platform with multiaxial foot up to 20 degrees and can be adjusted to provide different instability degrees. It has one static level and twelve varied, and it can be free to move in the anterior-posterior and medial-lateral directions at the same time, where 1 is the least stable adjustment and 12 the most stable one. Tests were performed in the static platform and then in the dynamic one (varying from the level 12 to 8 ). The subjects were instructed to keep a stable and comfortable bipedal posture, with arms hanging and barefoot, with the heels at the points D6 (left side) and D16 (right side), and the angle between the second and third toes of each foot was written down so that the same position can be repeated in the reevaluations. It was performed tests of Global, medial-lateral and anterior-posterior angular oscillation of the individual's center of gravity that measure the Postural Stability and Fall Risk. Each test was repeated 3 times, during 20 seconds, with a 10-second-break after each repetition and it was found a mean for the calculation. ${ }^{(15)}$

Functional mobility evaluated through the Timed Up and Go Test consisted of standing up from a chair $(45 \mathrm{~cm}$ of height from the seat) without using the arms; walking safely for three meters in a straight line as fast as possible; turning around a cone and going back sitting again on the chair so the time spent to perform the test would be registered. ${ }^{(16)}$ The test was

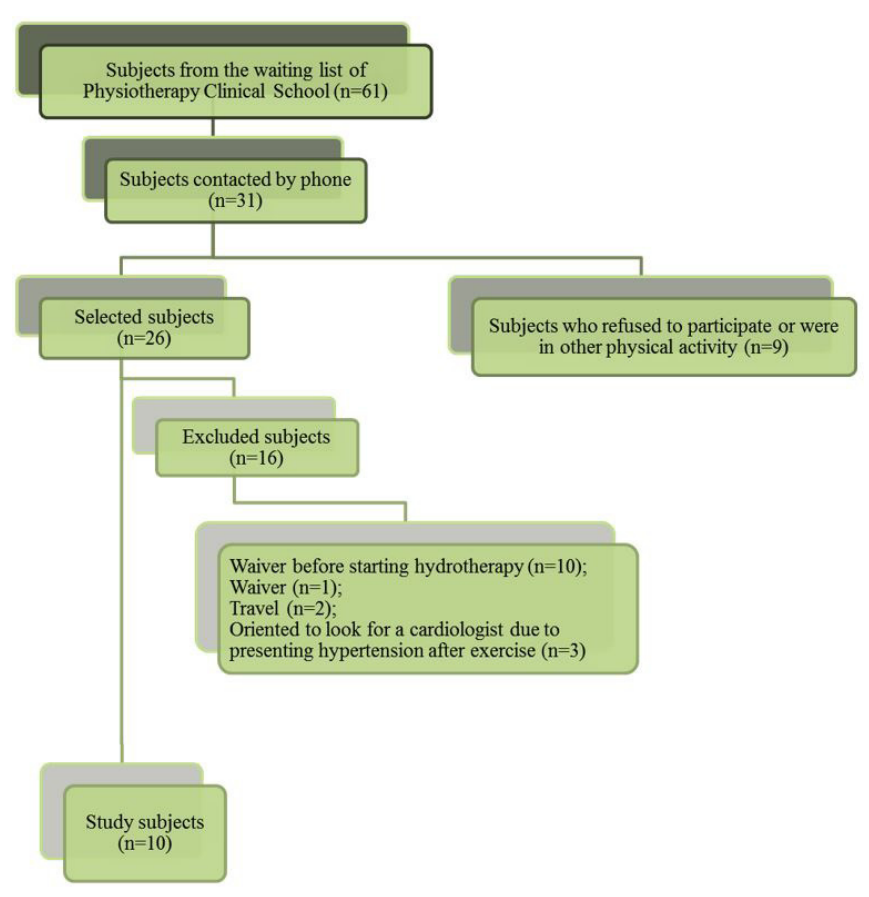

Figure 1. Flow diagram of study subjects 
explained and demonstrated by the examiner, and then the subjects performed it just to be more familiar with what they were supposed to do, and after that they performed again to register the time spent.

The Berg Balance System ${ }^{(17)}$ verified the dynamics balance through 14 common tasks in daily activities, using: a stopwatch, a tape measure, a chair and a 2-step-ladder. All tasks were demonstrated and explained by the examiner. Grading could vary from 0 to 56 points. There are 5 alternatives that vary from 0 to 4 points for each item.

\section{Treatment Protocol}

The intervention was conducted in the therapeutic pool of the Physiotherapy Clinical School in UFPB. Vital signs were verified before and after the exercises in order to check the feasibility of performing the exercises with no statistical purposes. The treatment protocol was adapted from Resende et al. ${ }^{(18)}$ aiming to improve postural control and reducing fall risk, and it had four stages, in this order: warm-up exercises; stretching; kinesiotherapy focused on static and dynamic balance; and relaxation. The exercises were done using tools such as trampoline, balance disc, step, ball, bats and floats (noodles) and the water depth level varied according to the activities. The training was conducted in 24 45-minute-sessions, twice a week, for a period of three months.

\subsection{Data analysis}

Data was analyzed through the Statistical Package for Social Sciences (SPSS - 20.0) software, in which the dependent variable was anterior-posterior (APD), medial-lateral (MLD) and global (GD) displacement, measured in radians (rad) in the dynamic and static Postural Stability the BS, dynamic and static Fall Risk the BS, the grading in the BBS and the time to perform the TUGT, while the independent variable was the experimental group.

After the Shapiro-Wilk test, the Anova was done for repeated measures with post hoc of Bonferroni for data regarding Dynamic Postural Stability the BS and the TUGT; the Friedman and the Wilcoxon for Static Postural Stability, Fall Risk the BS and BBS data. Results were classified as pre-intervention (pre), after 12 (post12) and 24 (post24) intervention sessions and a $\alpha=0.05$ significance level was considered.

\section{RESULTS}

It was observed that $90 \%$ of the subjects mentioned having already fell down; $70 \%$ admitted noticing some problems or difficulties to walk, however, only $40 \%$ reported fear of falling. All of them were gait independents and did not use any auxiliary tool, but $20 \%$ were afraid of going out of their homes alone.
The Anova test for repeated measured showed that the Global (DGPS), Anterior-posterior (DAPPS), Medial-lateral (DMLPS) Postural Stability score evaluated in the dynamic platform showed significant difference $(P<0.05)$ after 24 intervention sessions and, in the TUGT, there was reduction in time, with statistically significant differences after 12 and 24 intervention sessions $(P<0.05)$, showing an improvement in functional mobility (Table 1 ).

The Friedman test revealed that only the variable related to Static Global Postural Stability (SGPS) presented significant difference $(P<0.05)$ at the moment Post24. Regarding the Static and Dynamic Fall Risk the BS evaluation results, no variable showed statistically significant change $(P>0.05)$ after intervention and, in the BBS, all of the subjects got the highest score at the end of the training, with statistically significant differences after the 12 and 24 intervention sessions $(P<0.05)$, showing an improvement in functional balance (Table 2 ).

\section{DISCUSSION}

According to the results in this study, there was significant improvement in dynamic postural stability regarding all variables. However, the static postural stability only improved in the global score, and concerning the fall risk analysis it was not observed any significant result.

Balance plays an important role in the independence for daily activities and it is present in static and dynamics situations. Balance deficit may lead elderly individuals to a voluntary isolation, exacerbating the clinical diagnosis and, as a consequence, it may increase the restrictions in the daily activities and make them lose functional independence, severely affecting their quality of life.

The study of Ghulyan, Paolino ${ }^{(19)}$ showed that dynamic postural control is hindered in people who fall down when compared to those who have never fallen. The difference between these two groups was found during disturbances that required automatic adjustments as well as in voluntary movements.

Most falls happen during dynamic activities, since they challenge balance, demanding more postural stability control and fast response to the displacement stimulus of the center of gravity regarding the base of support. ${ }^{(20,21)}$

The results of the Postural Stability test of Balance System confirmed that there was a significant improvement in global displacement score in the static platform, and in the anterior-posterior, medial-lateral and global scores in the dynamic platform. According to Hilliard et al. $\left({ }^{(21)}\right.$ there is evidence that the effects of aging on balance can be accentuated in MLD, and most studies on falls prevention focus on balance and gait performance involving the APD. Aydoğ et al. ${ }^{(20)}$ evaluated comparatively dynamics balance in two groups using the BS - subjects with and without rheumatoid arthritis. They identified that dynamic balance is hindered in patients with rheumatoid arthritis, especially in the MLD control, which seemed to be poorer in the evaluation. 
Similar results were found by Melzer et al. ${ }^{(22)}$ since elderly individuals who had more MLD oscillations showed greater fall risk. These researches did not do the intervention in order to improve balance deficit, but they show that there is an imbalance in the APD as well as in the MLD of elderly people with balance deficit, and that a few of them evaluate the MLD oscillations. The non-evaluation of this variable contributed to the non-development of effective therapeutic strategies to restore/maintain elderly individuals' balance.

The Timed Up \& Go Test showed reduced time to run the test significantly, expressing improvement in functional mobility of the subjects in this study. The results of Alikhajeh et al. ${ }^{(23)}$ who also used TUGT to evaluate balance in 28 sedentary elderly individuals, divided into two groups - one experimental and the other control - before and after hydrotherapy, are in accordance with the findings in this study. 24 sessions were held, however, it lasted for 2 months, three times a week. Similarly, there was a significant reduction in time to perform the test.

The pre and post aquatic therapy evaluation by Berg Balance System found that there was improvement in balance after intervention in all elderly individuals, and it was observed that they reached top balance, respecting the BBS limits. These results are similar to the ones found by Hosseini et al. ${ }^{(24)}$ in which BBS was used to verify balance improvement before and after aquatic training for eight weeks; the subjects did not reach the highest score but showed significant score increase in the scale. It can be suggested that the failure in reaching the highest score may be due to the fact that the group shows a higher age average when compared to this study, so it is necessary to have an intervention period longer than 24 sessions.

Resende et al. ${ }^{(18)}$ and Kieffer et al. ${ }^{(25)}$ got positive results with 12 training sessions. In this study, at the first re-evaluation after 12 sessions, it was also seen a significant improvement according to TUGT and BBS. However, in the balance platform (BS) these results were only significant after 24 training sessions. It may be due to the fact that BS presents more reliable values to verify the displacement of the center of gravity in relation to its base of support. ${ }^{(15)}$ The longer neuromuscular response time may be justified by the necessity of the neuromuscular system to adapt itself to the physical exercise, since elderly individuals already show a reduced physical capacity. This finding leads to the fact that longer and more frequent interventions may be more effective to the recovery of elderly individuals, since balance is a complex mechanism, and the neuromuscular system needs time to adapt itself and respond effectively to the training.

Table 1. Mean, standard deviation (SD) and comparisons of means from variables of the Dynamic Postural Stability the BS and of the Timed Up \& Go Test in pre-intervention, post-intervention 12 and 24 sessions in the Anova test for repeated measures and the Post Hoc de Bonferroni.

\begin{tabular}{|c|c|c|c|c|c|c|c|c|c|}
\hline \multirow{3}{*}{ VARIABLES } & \multirow{2}{*}{ Pre } & \multirow{2}{*}{ Post12 } & \multirow{2}{*}{ Post24 } & \multicolumn{6}{|c|}{ Comparison of evaluations } \\
\hline & & & & \multicolumn{2}{|c|}{ Pre $x$ Post12 } & \multicolumn{2}{|c|}{ Post12 x Post24 } & \multicolumn{2}{|c|}{ Pre x Post24 } \\
\hline & $\bar{X} \pm S D$ & $\bar{X} \pm \mathrm{SD}$ & $\bar{X} \pm \mathrm{SD}$ & Dif Mean & $P$ & Dif Mean & $P$ & Dif Mean & $P$ \\
\hline DGPS & $1.59 \pm 0.42$ & $1.37 \pm 0.33$ & $1.10 \pm 0.40$ & 0.22 & 0.20 & 0.27 & 0.08 & 0.49 & 0.01 \\
\hline DAPPS & $1.19 \pm 0.34$ & $0.89 \pm 0.26$ & $0.77 \pm 0.33$ & 0.30 & 0.06 & 0.12 & 0.78 & 0.42 & 0.01 \\
\hline DMLPS & $0.86 \pm 0.26$ & $0.82 \pm 0.27$ & $0.63 \pm 0.22$ & 0.04 & 1.00 & 0.19 & 0.10 & 0.23 & 0.04 \\
\hline TUGT & $9.54 \pm 1.47$ & $8.67 \pm 1.18$ & $7.47 \pm 0.79$ & 0.88 & 0.01 & 1.20 & 0.01 & 2.07 & 0.01 \\
\hline
\end{tabular}

Legend: DGPS - Dynamic Global Postural Stability; DAPPS - Dynamic Anterior-posterior Postural Stability; DMLPS - Dynamic Medial-lateral Postural Stability; TUGT - Timed Up \& Go Test.

Table 2. Comparisons of the Static Postural Stability, Fall Risk the BS and Berg Balance Scale variables average in pre-intervention, post-intervention 12 and 24 sessions in the testing of Friedman and Wilcoxon.

\begin{tabular}{|c|c|c|c|c|}
\hline \multirow{3}{*}{ VARIABLES } & \multirow{3}{*}{ Friedman Value of $\boldsymbol{P}$} & \multicolumn{3}{|c|}{ Comparison of evaluations } \\
\hline & & \multicolumn{3}{|c|}{ Wilcoxon Value of $P$} \\
\hline & & Pre $x$ Post12 & Post12 x Post24 & Pre x Post24 \\
\hline SGPS & 0.03 & 0.23 & 0.15 & 0.02 \\
\hline SAPPS & 0.06 & 0.16 & 0.23 & 0.05 \\
\hline SMLPS & 0.48 & 0.93 & 0.49 & 0.27 \\
\hline DFR & 0.34 & 0.95 & 0.08 & 0.17 \\
\hline SFR & 0.10 & 0.65 & 0.02 & 0.11 \\
\hline BBS & 0.01 & 0.02 & 0.07 & 0.02 \\
\hline
\end{tabular}

Legend: SGPS - Static Global Postural Stability; SAPPS - Static Anterior-posterior Postural Stability; SMLPS - Static Medial-lateral Postural Stability; DFR - Dynamic Fall Risk; SFR - Static Fall Risk; BBS - Berg Balance Scale. 
Many studies show that training in therapeutic pool generate statistically significant results to the improvement of neuromuscular performance and postural stability control capacity, reducing balance deficit, and it can be recommended as an efficient intervention tool. ${ }^{(18,23-27)}$ However, the intervention program and the evaluation used by these authors are different. In addition to subjective tests, it was used the BS, which is considered a more reliable program to verify the displacement of the center of gravity during movement and when static, ${ }^{(15)}$ so these factors hinder the static comparison.

The authors Abbasi et al. ${ }^{(26)}$ and Hosseini et al. ${ }^{(24)}$ besides proving a significant improvement in postural balance in sedentary elderly people, also verified that after 4, 6 and 8 weeks without training, elderly individuals kept the gains obtained and the effects of aquatic training.

A possible explanation for the fact that aquatic environment is propitious to exercise and promotes balance recovery is directly linked to water physical properties associated with the effects of regular physical training.

The main limitations of this study were the impossibility of creating a control group, the fact that it was not possible to randomly select the subjects for the experimental group and the lack of similar studies, since it narrows the discussion of the presented data. It is suggested that, in further studies, these mentioned aspects be taken into account.

\section{CONCLUSION}

This study showed that regular physical training in water provides elderly individuals with a significant improvement in dynamic balance capacity, showing to be an effective source for keeping and improving balance capacity in the elderly population. Moreover, the training protocol in aquatic environment was proved to be safe, and it also provides elderly individuals with conditions to be challenged to go beyond their stability limits, not fearing the consequences of a fall.

\section{AUTHORS' CONTRIBUTIONS}

ACCRC: Data analysis and scientific writing; HHS: Data analysis; JJAF: Assessing the subjects and scientific writing; PRA: Orientation of the project and scientific writing; TMSR: Data collection, tabulation and data analysis; TMA: Development and writing of the research project, data collection, tabulation, data analysis and scientific writing.

\section{CONFLICTS OF INTEREST}

The authors declare that they have no conflict of interest.

\section{AUTHOR DETAILS}

${ }^{2}$ Universidade Federal da Paraíba (UFPB), João Pessoa (PB), Brazil

\section{REFERENCES}

1. Veras R. Envelhecimento populacional contemporâneo: demandas, desafios e inovações. Rev. Saúde Pública. 2009;43(3):548-554.

2. Instituto Brasileiro de Geografia e Estatística [IBGE] Censo demográfico: Brasil; 2010.
3. Gazzola JM, Perracini MR, Ganança MM, Ganança FF. Fatores associados ao equilíbrio funcional em idosos com disfunção vestibular crônica. Rev Bras Otorrinolaringol. 2006;72(5):683-690.

4. Rogers MW, Mille M-L. Lateral stability and falls in older people. Exerc Sport Sci Rev. 2003;31(4):182-187.

5. Gawryszewski VP, Jorge MHPM, Koizumi MS. Mortes e internações por causas externas entre os idosos no Brasil: o desafio de integrar a saúde coletiva e atenção individual. Rev Assoc Méd Bras. 2004;50(1):97-103.

6. Hawk C, Hyland JK, Rupert R, Colonvega M, Hall S. Assessment of balance and risk for falls in a sample of community-dwelling adults aged 65 and older. Chiropractic \& Osteopathy. 2006;14(3):1-8.

7. Romero $\mathrm{CA}$, Iturbe $\mathrm{AG}$, Gil CL, Lesende IM, Santiago AL. Actividades preventivas en los ancianos. Aten Primaria. 2001;28(2):161-190.

8. Duarte M, Freitas SMSF. Revisão sobre posturografia baseada em plataforma de força para avaliação do equilíbrio. Rev. Bras Fisioter. 2010;14(3):183-192.

9. Maciel ACC, Guerra RO. Prevalência e fatores associados ao déficit de equilíbrio em idosos. R Bras Ci e Mov. 2005;13(1):37-44.

10. Almeida APPV, Veras RP, Doimo LA. Avaliação do equilíbrio estático e dinâmico de idosas praticantes de hidroginástica e ginástica. Rev. Bras Cineantropom Desempenho Hum. 2010;12(1):55-61.

11. Caromano FA, Candeloro JM. Fundamentos da hidroterapia para idosos. Arq Ciên da Saúde Unipar. 2001;5(2):187-195.

12. Rabelo RJ, Bottaro M, Oliveira RJ, Gomes L. Efeitos da natação na capacidade funcional de mulheres idosas. R Bras Ci e Mov. 2004;12(3):57-60.

13. Padoin PG, Gonçalves MP, Comaru T, Silva AMV. Análise comparativa entre idosos praticantes de exercício físico e sedentários quanto ao risco de quedas. O Mundo da Saúde. 2010;34(2):158-164.

14. Biodex Medical Systems, INC. Reference manual-Overview. United States of America. 2005:1-12.

15. Parraca JA, Olivares PR, Baeza AC, Aparicio VA, Adsuar JC, Gusi N. Testretest reliability of Biodex Balance SD on physically active old people. J Hum Sport Exerc. 2011;6(2):444-451.

16. Podsiadlo D, Richardson S. The timed "up \& go": A test of basic functional mobility for frail elderly persons. J Am Geriatr Soc. 1991;39(2):142-8.

17. Miyamoto ST, Jr. IL, Berg KO, Ramos LR, Natour J. Brazilian version of the Berg balance scale. Braz J Med Biol Res. 2004;37(9):1411-1421.

18. Resende SM, Rassi CM, Viana FP. Efeitos da hidroterapia na recuperação do equilíbrio e prevenção de quedas em idosas. Rev. Bras Fisioter. 2008;12(1):57-63.

19. Ghulyan V, Paolin M. Comparative study of dynamic balance in fallers and non-fallers. Fr ORL. 2005;88:89-96.

20. Aydoğ E, Bal A, Aydoğ ST, Çakci A. Evaluation of dynamic postural balance using the Biodex Stability System in rheumatoid arthritis patients. Journal ILAR. 2006;25:462-467.

21. Hilliard MJ, Martinez KM, Janssen I, Edwards B, Mille M-L, Zhang Y et al. Lateral balance factors predict future falls in community-living older adults. Arch Phys Med Rehabil. 2008;89(9):1708-1713.

22. Melzer I, Benjuya N, Kaplanski J. Postural stability in the elderly: a comparison between fallers and non-fallers. Age and Ageing. 2004;33(6):602-607.

23. Alikhajeh $Y$, Hosseini SRA, Moghaddam A. Effects of hydrotherapy in static and dynamic balance among elderly men. Procedia Soc Behav Sci. 2012;46:2220-2224. 
24. Hosseini SS, Mirzaei B, Panahi M, Rostamkhany H. Effect of aquatic balance training and detraining on neuromuscular performance, balance and walking ability in healthy older men. Middle-East J Sci Res. 2011;9(5):661666.

25. Kieffer HS, Lehman MA, Veacock D, Korkuch L. The effects of a short-term novel aquatic exercise program on functional strength and performance of Older Adults. Int J Exerc Sci. 2012;5(4):321-333.
26. Abbasi A, Sadeghi H, Tabrizi HB, Bagheri K, Ghasemizad A, Asl AK. Effect of whole body vibration, aquatic balance and combined training on neuromuscular performance, balance and walking ability in male elderly able-bodied individual. World Appl Sci J. 2001;15(1):84-91.

27. Devereux K, Robertson D, Briffa NK. Effects of a water-based program on women 65 years and over: a randomised controlled trial. Aust J Physiother. 2005;51:102-108. 